\title{
Mobile health for cancer in low to middle income countries: Priorities for research and development
}

\author{
Isaac Holeman, BA, MPhil \\ Gates Cambridge Scholar, Judge Business School, University of Cambridge \\ Fellow, Global Health Academy, University of Edinburgh \\ Cofounder, Medic Mobile \\ King's College, Cambridge, UK CB2 1ST \\ Correspondence address: isaac@medicmobile.org
}

Jay Evans, BA, MSc

Asia Regional Director, Medic Mobile

Lecturer, Global Health Academy, University of Edinburgh

200 Halleck Street San Francisco, California, USA 94129

Dianna Kane, BA, MPhil

Senior Designer, Medic Mobile

200 Halleck Street San Francisco, California, USA 94129

\section{Liz Grant, MA, PhD}

Deputy Director, Global Health Academy, University of Edinburgh, Centre for Population Health Sciences, University of Edinburgh Medical School Teviot Place, Edinburgh UK EH8 9AG

\section{Claudia Pagliari, BSc, PhD, FRCPE}

Director of Global eHealth, Convener eHealth Interdisciplinary Research Group, Global Health Academy \& Centre for Population Health Sciences, University of Edinburgh Medical School Teviot Place, Edinburgh UK EH8 9AG

\section{David Weller, MBMS, MPH, FRCGP, FRCPE, PhD}

James MacKenzie Professor of General Practice

Centre for Population Health Sciences, University of Edinburgh Medical School

Teviot Place, Edinburgh UK EH8 9AG

\section{Acknowledgements:}

We declare no sources of financial support for the writing of this editorial. We thank Medic Mobile for the use of illustrations that formed the basis of Figure 1, and the teams at Medic Mobile and St Gabriel's Hospital for supporting the demonstration projects discussed in this editorial.

This is the author owned, accepted version of this manuscript. The version of record was published in October 2014 and is owned by the publisher. It may be downloaded free of charge at http://onlinelibrary.wiley.com/doi/10.1111/ecc.12250/abstract 


\begin{abstract}
Many current global health opportunities have less to do with new biomedical knowledge than with the coordination and delivery of care. While basic research remains vital, the growing cancer epidemic in countries of low and middle income warrants urgent action - focusing on both research and service delivery innovation. Mobile technology can reduce costs, improve access to health services, and strengthen health systems to meet the interrelated challenges of cancer and other noncommunicable diseases. Experience has shown that even very poor and remote communities that only have basic primary health care can benefit from mobile health (or 'mHealth') interventions. We argue that cancer researchers and practitioners have an opportunity to leverage mHealth technologies that have successfully targeted other health conditions, rather than reinventing these tools. We call for particular attention to human centred design approaches for adapting existing technologies to suit distinctive aspects of cancer care and to align delivery with local context - and we make a number of recommendations for integrating mHealth delivery research with the work of designers, engineers and implementers in large-scale delivery programmes.
\end{abstract}

Keywords: delivery research, human centred design, global health, mHealth, service delivery innovation, developing countries; cancer; community based health workers; low income countries, innovation, mobile health, technology, service delivery, primary care, cancer and eHealth 


\section{The mHealth opportunity}

In 2011 the World Health Organization (WHO) proclaimed that mobile technology for health, or mHealth, has the "potential to transform the face of health service delivery across the globe" (Kay et al. 2011). More than $90 \%$ of the world's population now lives within reach of a mobile phone signal (ITU 2010), vastly exceeding the proportion of the population with access to basic health care. While some have highlighted the potential of mHealth interventions as general health systems strengthening tools (Labrique et al. 2013), most trials have evaluated technologies that were built from scratch for use in specific health areas such as HIV adherence (Lester et al. 2010) or malaria control (Zurovac et al. 2011). There is a need to build on existing mHealth innovations with a focus on cancer care, particularly in low and middle-income countries.

Cancer survival rates vary greatly across countries (Coleman 2014) and the burden of disease in low-income countries is growing rapidly (Vineis \& Wild 2014). Countries of low and middle income now account for $80 \%$ of the disability-adjusted life-years lost worldwide to cancer (Sloan, 2007), yet are estimated to receive only 5\% of global resources for cancer (Ngoma, 2006). Demographic shifts and economic trends associated with an increase in behavioural risk factors, such as tobacco use and unhealthy diets, are exacerbating the burdens of cancer and other noncommunicable diseases in these countries (Alwan et al. 2010).

Farmer et al (2010) have described a number of projects demonstrating that cancer care is challenging yet possible in even the poorest communities. For example, national health insurance programmes in Mexico and Colombia have demonstrated that coordinated financing and procurement can reduce prices and expand access. Successful programmes in Malawi, Rwanda and Haiti have shown that effective diagnosis and treatment can be introduced in poor and rural areas that lack specialised services. What is more, sharing tasks with Community Health Workers (CHWs) can improve cancer care while broadly benefiting primary health systems. Scaling up such efforts will require innovative approaches to service delivery that reduce costs, improve access, and strengthen health systems to meet the interrelated challenges of cancer and other noncommunicable diseases.

\section{An Example of mHealth service delivery innovation in a low-resource setting}

Some regions of the world have very little in the way of primary prevention, cancer screening, and basic cancer treatments. Often there are very significant delays in cancer diagnosis due to economic, social and cultural challenges (Pati et al. 2014). While these constraints are considerable, experience 
in other health areas has shown that even very poor and remote communities with only basic primary health care provision can benefit from mHealth interventions.

As an example, in 2008, the nonprofit technology company Medic Mobile began a partnership with St. Gabriel's Hospital in Malawi. Like many low-income countries, Malawi's severe health worker shortage is exacerbated by the urban concentration of highly trained health professionals and the geographic dispersion of a rural population. Of the country's 27 districts, four had no doctors at all, five had fewer than one nurse per facility, and 15 had fewer than 1.5 nurses per facility as of 2004 (Evans et al. 2006). In such settings, involving a broader array of health workers can improve health outcomes while more efficiently leveraging limited health resources. Community-based lay health workers (CHWs), family members and non-medical staff can partner with clinicians, bringing new competencies and dramatically improving access to care. However, their training is minimal, they often lack the equipment or expertise to deal with complex cases and they can become just as disconnected from expert advice as the patients they serve.

To bridge this gap, Medic Mobile equipped an initial group of $75 \mathrm{CHWs}$ with mobile phones and trained them to exchange text messages with a nurse at the Malawian district hospital (Image 1). Many of the CHWs had never used a mobile phone, and the nurse had not used a laptop before the program began. The CHWs soon were exchanging messages for a variety of purposes, some of which were planned in advance and some of which only emerged in practice. A retrospective analysis of the first six months of the pilot found that common uses included patient referrals, reporting symptoms, requesting supplies and requesting help or advice (Mahmud et al. 2010). Most of the messages concerned patients with cancers receiving community-based palliative care, or patients with HIV or Tuberculosis.

The program at St Gabriel's is still operating independently, and now is among the older continuously active, locally managed mHealth programs in Africa. This same approach to field communications has since been replicated repeatedly throughout Malawi and in other countries. An independent survey of CHWs at one replication site found that text messaging was less expensive and more than an order of magnitude faster than walking, bicycling or taking public transit— the most common alternative means of reaching supervisors for support or reporting (Lemay et al. 2012).

For the CHWs, the value of this tool was less about disease-specific content and more about efficiency, for example not having to walk or ride a bicycle long distances to the hospital to deliver 
reports or ask for basic advice or support. While other demonstration projects have focused exclusively on cancer care, this initiative illustrates how mHealth interventions can improve cancer treatment and palliation in ways that broadly improve the delivery of primary health care.

\section{Can mHealth interventions improve cancer outcomes in low-resource settings?}

In brief, little directly relevant evidence is available and rigorous studies of mHealth interventions for cancer programmes in poor countries are scarce. However, there is merit in drawing on the evidence base generated in and for other health areas. Figure 1 portrays the basic structure of a typical low-income country health system and several points at which mobile technology could facilitate prevention, screening, early diagnosis, treatment or palliative care. There is some evidence to underpin this model; for example, a systematic review by Free et al. (2013) demonstrates that mobile technology interventions for smoking cessation have proven effective in high income settings, and calls for their effectiveness in low income settings to be established (see Figure 1, part 1). Behaviour change communication interventions related to smoking or other risk factors are often text message-based, a technology with the advantages of being accessible on any phone, asynchronous (SMS can be read later even if a recipient's phone is out of battery or has no connectivity at the time they are sent), and amenable to automated bulk delivery. Employing similar bulk-SMS technology, a trial in Kenya found that text message reminders increased health workers' adherence to paediatric malaria treatment protocols by $23 \%$ over the course of six months, and that the effects were retained six months after the intervention ended (Zurovac et al. 2011). Similar technology might be used in tandem with in-person training to reinforce cancer care practices among remote health workers (see Figure 1, part 2).

A number of mHealth pilots involve community-based case finding (see Figure 1, part 3). In some projects CHWs are trained to make a preliminary check for diseases such as Tuberculosis, and use generic SMS or forms-based data collection tools to refer patients to higher care or request assistance from a mobile testing unit (Mahmud et al. 2010). A trial in Bangladesh found that CHWs were more effective at breast health promotion when guided through the screening process by a smartphone application (Ginsburg, 2014). In a project in the Indian state of Karnataka, CHWs were equipped with smartphones that enabled them to send images, audio, location data and text, so that clinicians could remotely screen individuals at-risk for oral cancer (GSMA 2012).

For screening and early diagnosis initiatives to be effective, there must be mechanisms in place to undertake basic functions such as recruitment, provision of test results (to patients and health care 
workers) and follow-up with further investigation and/or treatment. Without this basic information infrastructure, screening and early diagnosis efforts are not only hindered, they also have the potential to do more harm than good. Better referral systems could accelerate this lengthy process and improve patient follow up (Figure 1, part 5). Approaches that leverage mobile technology are being used, for example, to expedite early infant diagnosis, referral and care for HIV in Zambia (Lambo et al. 2011).

Once patients are enrolled in an mHealth programme, a variety of technologies exist to manage patient care pathways, for example scheduling and delivering notifications concerning upcoming or recently missed appointments. Some involve the use of feature or smartphones that enable CHWs to keep patient records and coordinate who to visit and when (Mhila et al. 2009). Other projects store patient records and schedules at a central hub, and send appointment notifications via text message or voice mails (e.g. Crawford et al. 2013). These messages might be sent directly to patients or to CHWs who then visit patient homes (Figure 1, part 4).

Collecting data on service statistics and the supply of drugs and other medical commodities can strengthen the supply chain and improve long-term planning efforts (Figure 1, part 6). This is particularly important for palliation because patients are less likely to be mobile enough to seek services at referral facilities and a disrupted supply of essential medicines such as morphine can lead to patient suffering and poor outcomes. An exemplar mHealth supply chain initiative is the SMS for Life project in Tanzania, in which malaria medicine stock-outs fell from $78 \%$ at week 1 to $26 \%$ at week 21 of the pilot (Barrington et al. 2010).

Finally, in cases where curative cancer treatment is not accessible (as is often the case in lowresource settings), good quality palliative care to relieve pain and minimize suffering is essential and often best provided in the community. In such cases, mobile technology has been used to strengthen links between facility-based experts and CHWs or patients and their family caretakers (Figure 1, part 7). In community-based palliative care settings, technologies that enable a supportive two-way dialogue, such as text messaging and phone calls, are often more appropriate than standardized and automated messaging (Grant et al. 2011).

It is important to note that individual phone ownership is often lower among vulnerable populations (UN 2014). Cancer control initiatives that rely too heavily on patients accessing and using mobile phones might exacerbate inequities that already shape many aspects of the patients' lives. In contrast, mHealth initiatives that equip community health workers with phones can take full 
advantage of the fact that $90 \%$ of the world's population now lives within reach of a mobile phone signal (ITU 2010). Opportunities exist across the cancer spectrum, from promoting preventative behaviours and prompt screening to curative therapy and palliative care. Numerous demonstration projects and some evidence suggest that mobile technologies including SMS, feature phones, smartphones and a variety of software applications can be used to improve health service delivery in low to middle income countries. While these programmes and studies do not provide an evidence base to justify immediate scale up of mHealth interventions in cancer control, they do provide a basis for future service development and highlight research priorities.

\section{Designing for local context}

Our call to build on existing mHealth interventions is based on the understanding that adapting these tools for cancer care is preferable to the development of analogous technologies and service delivery approaches from scratch. Nevertheless, it must not be assumed that a technology used successfully in malaria control will also prove effective in cancer control, or that the use of smartphones will always be beneficial when evidence of effectiveness is limited to a narrow range of settings. There is growing recognition that mHealth initiatives are best thought of as complex interventions (PLOS Medicine Editors 2013) because their success typically hinges on changes in human behaviour and because they evolve not only from project to project but even during the course of implementation. Complex innovations do not automatically successfully translate to new contexts, for a host of socio-technical reasons such as different existing care processes, cultural resistance and lack of technological fit. Repurposing a product from one disease area or setting to another therefore calls for the application of adaptive design principles.

While designers traditionally focused on enhancing the functionality and appeal of physical products and software, the same toolkit is increasingly driving innovation around more complex challenges such as healthcare delivery (Brown \& Wyatt 2010). There is no single recipe for human centred design (Kling \& Star 1998), but such approaches typically prioritize user perspectives and observation of the social or organizational circumstances in which a new product will be used, rather than relying on developers' preconceptions or rigid procurement briefs (Pagliari 2007). Common techniques include fostering empathy by spending time in the company and circumstances of the people who will experience the intervention, using diverse prototypes to explore and develop possible intervention adaptations, and actively engaging program participants to iteratively improve 
the proposed intervention. The value of engaging beneficiary communities in collectively developing and implementing interventions is not unique to mHealth. For example, a review of cancer control studies among indigenous people found that they were more likely to lead to improved outcomes when communities were genuinely involved at all stages of the intervention and/or research process, including identifying the priority or need through to the dissemination of results (Miller 2012). Such activities often reveal new opportunities as well as unanticipated barriers that require interventions to be adapted before they can proceed effectively in a particular local community.

User centred research and development have long been practiced in high income countries (Waller 2006), and more recently have featured prominently in international development initiatives funded by agencies such as the Bill and Melinda Gates Foundation (IDEO 2009) and the UK Department For International Development (IDEO 2014). It is timely to explore how human centred design might improve cancer care and control initiatives in countries of low and middle income. The gap as we see it has less to do with developing new design principles than it does with applying them in the novel context of mHealth interventions for cancer care and control in low to middle income countries.

\section{mHealth Delivery Research}

The importance of engineering and design expertise in mHealth initiatives introduces challenges related to interdisciplinary collaboration with health services managers and researchers. Research demonstrating important impacts of mHealth interventions has often evaluated technologies that are insufficiently robust to merit widespread adoption (e.g. Lester et al. 2010; Zurovac et al. 2011), whilst the designers and implementers of successful mHealth projects often lack the skills to robustly evaluate their impacts (Nurmatov 2014). Thus, despite a plethora of mHealth projects and a vast pool of experiential learning amongst practitioners, medical researchers continue to call for a more rigorous evidence base to justify scale-up (Tomlinson et al. 2013). This trend is not unique to mHealth; similar barriers to interdisciplinary collaboration have been observed in the broader field of eHealth (Pagliari 2007).

Part of the problem lies in the disparate jargons, priorities, and operational constraints of these diverse professions. As has been noted elsewhere (Pagliari 2007), this pattern of working in parallel silos might be addressed by developing mutual awareness of, and respect for, each others' methods, 
epistemologies and contextual drivers. Such awareness can only develop through long-term collaboration and in the context of mutual trust. Thus we urge journal editors and agencies commissioning research and development in this area to favour for funding, review and publication, research that reflects long-term and meaningful engagement with designers, implementers, managers, and community members. Such interdisciplinary collaborations should not begin and end with a single demonstration project or clinical trial; at best they will begin with initial prototyping in a specific national health care system and proceed to robust evaluation and implementation at scale in the same setting. The United Kingdom Medical Research Council's guidance regarding complex interventions (Craig et al. 2008) is also highly relevant to the development, implementation and evaluation phases of this process. Research generated in this manner is most likely to address the needs and constraints of mHealth programmes in settings of poverty and high burdens of disease. Similarly, technologies developed through such collaborations are more likely to be meaningfully user-informed, fit for context, and of demonstrated value.

\section{Conclusion}

Many contemporary global health opportunities have less to do with new biomedical knowledge than with how we coordinate and deliver care, and how we repurpose established solutions for new problems, using expertise from local health workers, patients and families. While basic research, drug discovery and efforts to reduce the cost of therapeutics remain vital, the growing cancer epidemic in poor countries merits a sense of urgency about what can be done now. Mobile technologies are being used to encourage preventative behaviours, create patient pathways to care and enhance the quality of services. Many of these tools are open source and freely available. Adapting these interventions to support cancer care and control will require well-funded and concerted efforts of engineers, designers, clinicians and public health managers and researchers. While there are notable challenges and potential pitfalls, a bold mHealth innovation, research and implementation agenda has great potential to address the interrelated challenges of cancer and other non-communicable diseases worldwide. 


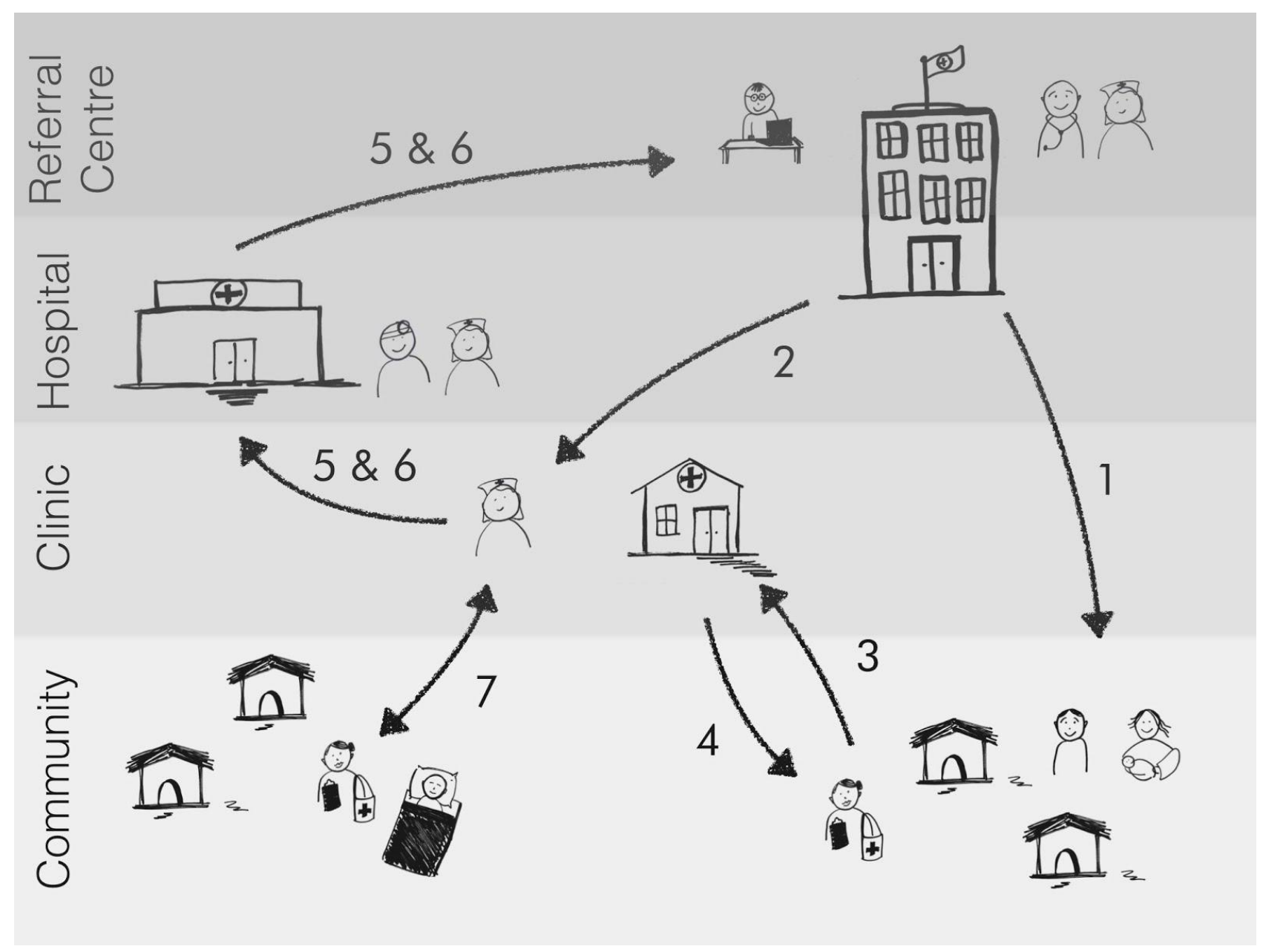

Figure 1: The basic structure of a typical low-income country health system and several points at which mobile technology could facilitate prevention, screening, early diagnosis, treatment or palliative care. See more illustrations of mHealth for cancer care at http://medicmobile.org/cancer

1) Behaviour change communication directed at patients and families

2) Behaviour change communication directed at health service providers

3) Screening and case finding

4) Tracing patients who have missed appointments

5) Referrals

6) Monitoring service statistics and supply chain of therapeutics

7) Post-treatment follow-up education, community-based palliative care support 


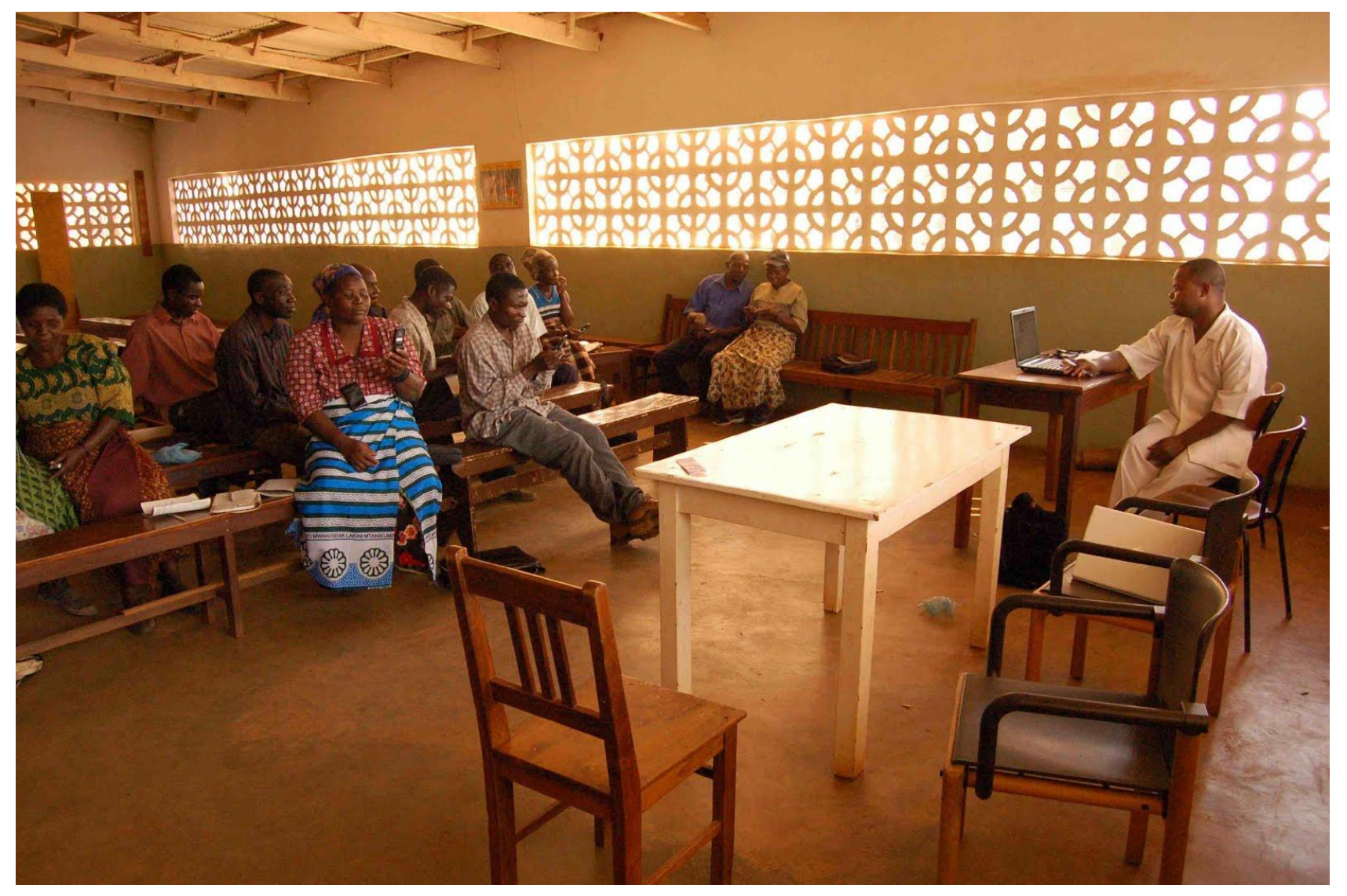

Image 1: Community Health Workers at St Gabriel's Hospital in Malawi attend an mHealth training session with Medic Mobile. 


\section{References}

Alwan, A. et al., 2010. Global status report on noncommunicable diseases, World Health Organization.

Barrington, J. et al., 2010. SMS for Life: a pilot project to improveanti-malarial drug supply management in ruralTanzania using standard technology. Malaria Journal, 9(1), p.298.

Brown, T. \& Wyatt, J., 2010. Design Thinking for Social Innovation. Stanford Social Innovation Review.

Coleman, M.P., 2014. Cancer survival: global surveillance will stimulate health policy and improve equity. The Lancet, 383(9916), pp.564-573.

Craig, P. et al., 2008. Developing and evaluating complex interventions: the new Medical Research Council guidance. BMJ, 337, pp.a1655-a1655.

Crawford, J. et al., 2013. SMS versus voice messaging to deliver MNCH communication in rural Malawi: assessment of delivery success and user experience. Global Health: Science and Practice, pp.35-46.

PLOS Medicine Editors, 2013. A Reality Checkpoint for Mobile Health: Three Challenges to Overcome. PLoS medicine, 10(2).

Evans, T. et al., 2006. Working Together for Health: The World Health Report, World Health Organization.

Farmer, P. et al., 2010. Health Policy. The Lancet, 376(9747), pp.1186-1193.

Free, C. et al., 2013. The effectiveness of mobile-health technology-based health behaviour change or disease management interventions for health care consumers: a systematic review. PLoS medicine, 10(1), p.e1001362.

Grant, L. et al., 2011. Palliative care making a difference in ruralUganda, Kenya and Malawi: three rapidevaluation field studies. BMC Palliative Care, 10(1), p.8.

GSMA, 2012. Sana Mobile Health Platform Case Study, Available at: http://www.gsma.com/connectedliving/sana-mobile-health-platform-case-study.

IDEO, 2009. Human Centered Design Toolkit 2nd ed., Available at: http://www.ideo.com/work/humancentered-design-toolkit.

IDEO ed., 2014. About Amplify, IDEO. Available at: https://openideo.com/content/about-amplify [Accessed June 2014].

ITU, 2010. World Telecommunication/ICT Development Report 2010, Geneva, Switzerland: International Telecommunications Union. Available at: http://www.itu.int/ITU-

D/ict/publications/wtdr_10/index.html.

Kay, M., Santos, J. \& Takane, M., 2011. mHealth: New horizons for health through mobile technologies: second global survey on eHealth, World Health Organization.

Kling, R. \& Star, S.L., 1998. Human Centered Systems in the Perspective of Organizational and Social Informatics. Computers and Society, pp.22-29.

Labrique, A.B. et al., 2013. mHealth innovations as health system strengthening tools: 12 common 
applications and a visual framework. Global Health: Science and Practice, 1(2), pp.160-171.

Lambo, N. et al., 2011. Project Mwana: Using Mobile Phones to Improve Early Infant HIV Diagnostic Services, Post-natal Follow-up and Care, UNICEF. Available at: http://www2.unicef.org:60090/equity/index_65314.html.

Lemay, N.V. et al., 2012. Reaching remote health workers in Malawi: baseline assessment of a pilot mHealth intervention. Journal of health communication, 17 Suppl 1(May 2012), pp.105-117.

Lester, R.T. et al., 2010. Effects of a mobile phone short message service on antiretroviral treatment adherence in Kenya (WelTel Kenya1): a randomised trial. Lancet, 376(9755), pp.1838-1845.

Mahmud, N., Rodriguez, J. \& Nesbit, J., 2010. A text message-based intervention to bridge the healthcare communication gap in the rural developing world. Technology and Healthcare, (18), pp.137-144.

Mhila, G. et al., 2009. Using Mobile Applications for Community-based Social Support for Chronic Patients, Dimagi. Available at: http://www.commcarehq.org/pdfs/mhila.pdf.

Miller J., Knott V.E., Wilson C. \& Roder, D., 2012. A review of community engagement in cancer control studies among Indigenous people of Australia, New Zealand, Canada and the USA. European Journal of Cancer Care, 21, 283-295.

Nurmatov, U.B. et al., 2014. The effectiveness of mHealth interventions for maternal, newborn and child health in low and middle-income countries: Protocol for a systematic review and meta-analysis. Journal of Global Health, 4(1), pp.1-8.

Pagliari, C., 2007. Design and Evaluation in eHealth: Challenges and Implications for an Interdisciplinary Field. Journal of Medical Internet Research, 9(2), p.e15.

Pati, S. et al., 2014. Care pathways, care experiences and barriers to treatment seeking in cancer : An exploratory study in Odisha, India. In press.

Tomlinson, M. et al., 2013. Scaling Up mHealth: Where Is the Evidence? PLoS medicine, 10(2), p.e1001382.

UN, 2014. United Nations E-Government Survey. Chapter 6: Bridging the Digital Divide, United Nations Department of Economic and Social Affairs.

Vineis, P. \& Wild, C., 2014. Global cancer patterns: causes and prevention. The Lancet, 383(9916), pp.549557.

Waller, A. et al., 2006. Participatory design of a text message scheduling system to support young people with diabetes. Health Informatics Journal, 12(4), pp. 304-318.

Zurovac, D. et al., 2011. The effect of mobile phone text-message reminders on Kenyan health workers' adherence to malaria treatment guidelines: a cluster randomised trial. Lancet, 378(9793), pp.795-803. 